\title{
Resumen de actividades del Comité Metropolitano de Tuberculosis: Selección de temas año 2008
}

\author{
Metropolitan Tuberculosis Committee. Summary of three presentations
}

Three presentations at the Metropolitan (Santiago de Chile) Tuberculosis Committee were selected to be published in this annual summary of 2008 activities. The three Tuberculosis issues described and discussed in this article are the following: 1) Comprehensive management of Tuberculosis and HIV in each control programs for tuberculosis and HIV; 2) Tuberculosis in childhood and 3) Auditing of tuberculosis deaths.

Key words: Tuberculosis, HIV, child tuberculosis, tuberculosis deaths.

\section{Resumen}

De las presentaciones hechas al Comité Metropolitano de Tuberculosis de Chile durante el año 2008, se seleccionaron 3 temas: 1) Manejo integrado de Tuberculosis y VIH en Programas de Control de la Tuberculosis y Programas de Control del VIH; 2) Tuberculosis Infantil y 3) Auditorías de Muerte en Tuberculosis".

Palabras clave: Tuberculosis, VIH, Tuberculosis infantil, Auditorías de muertes.

\section{Manejo integrado de Tuberculosis y VIH en Programas de Control de la Tuberculosis y Programas de Control del VIH}

Drs. Carlos Peña, Manuel Zúñiga,

Paulina Ramonda y EU. Zulema Torres,

Equipo de trabajo de los Programas de Control de la Tuberculosis.

Dr. Carlos Beltrán y E.U. Gloria Berríos, CONASIDA.

Antecedentes: La situación actual de la tuberculosis (TBC) es producto de un manejo programático centrado en la detección de los casos que trasmiten la enfermedad en la comunidad y en la aplicación de una terapia altamente eficaz. Estas actividades se desarrollan a través de una normativa ministerial con control de las actividades y una gratuidad garantizada de las acciones.

Un Programa de Control de la Tuberculosis eficaz y sostenido ha logrado una notable reducción de la incidencia de la TBC en Chile, la cual se concentra actualmente en los denominados grupos de riesgo, entre los que se destacan los pacientes VIH (personas viviendo con el VIH= PVVIH). Por otra parte, la infección VIH ha estabilizado su incidencia en Chile a través de un manejo preventivo organizado y de una tera- pia altamente efectiva y gratuita (terapia antirretroviral= TARV), lo que ha permitido una mejor calidad de vida y mayor sobrevida para estos pacientes.

Bases de la integración de ambos programas: Las PVVIH son susceptibles a enfermar de TBC según el grado de deterioro inmunológico que presentan (dependiendo del recuento de linfocitos CD4), pero la enfermedad se puede presentar en cualquier estadio de la infección VIH. Además, la TBC incrementa el deterioro inmunológico en los pacientes PVVIH. El diagnóstico de infectados VIH en pacientes con TBC coloca a estos casos en etapa de SIDA, con requerimiento de TARV.

Magnitud de la coinfección: No se conoce con exactitud debido a falta de estudios adecuados, pero de acuerdo a datos del Registro Nacional de TBC se estima cercana al $5 \%$.

Manejo integrado: El manejo integrado de ambas enfermedades nos ofrece la oportunidad de hacer un diagnóstico oportuno de la TBC en PVVIH y de detectar la infección VIH en pacientes TBC.

\section{Componente programático para PVVIH}

Paciente que ingresa a programa VIH/SIDA debe ser sometido a detección y prevención de $T B C$ (Figura 1). 


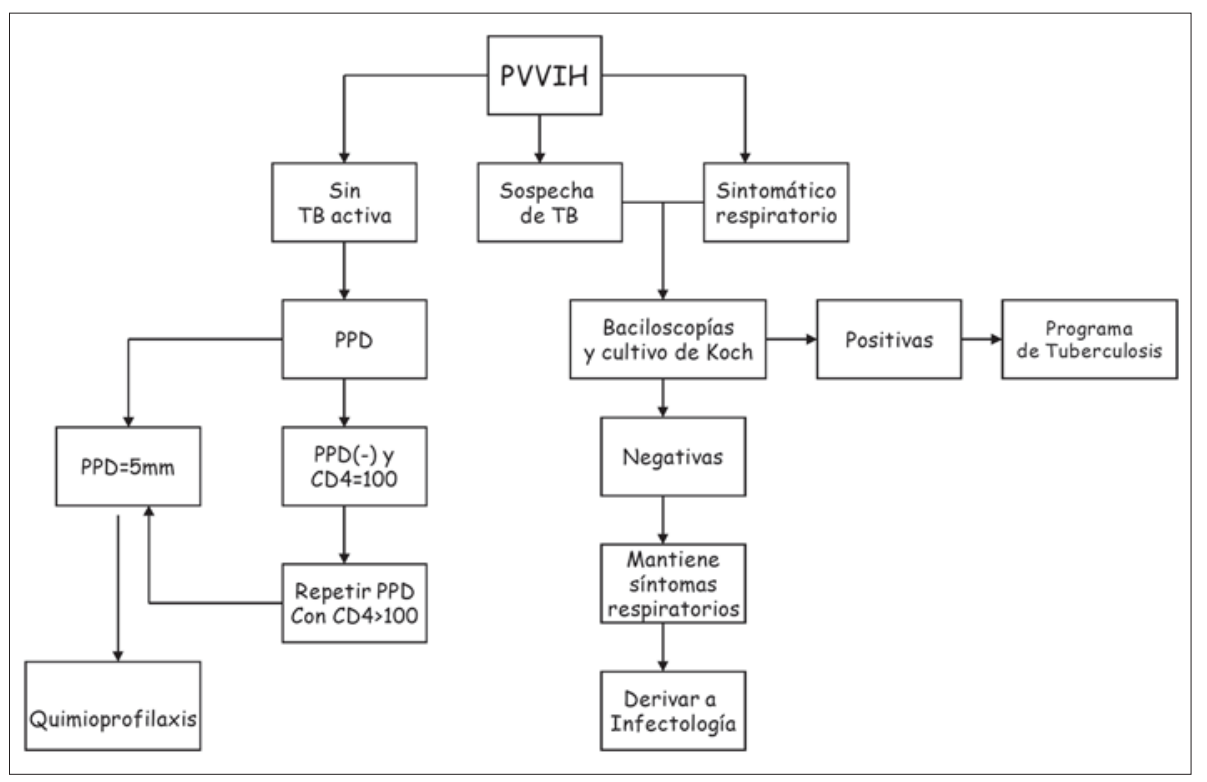

Figura 1. Flujograma para prevención y diagnóstico de tuberculosis (TB) en personas viviendo con VIH (PVVIH). Ver texto.

a) Dos baciloscopias con un cultivo de Koch en sintomáticos respiratorios

b) Radiografía de tórax

c) PPD con 2 UI de PPD 23 RT intradérmico, realizado en su servicio de salud o centro de referencia, por personal acreditado en este examen. Una induración cutánea $\geq 5 \mathrm{~mm}$ se considera positiva, pero debe ser interpretada de acuerdo al estado inmune celular, ya que producto del deterioro del recuento linfocitario ocasionado por el VIH, fármacos (prednisona) o por un deterioro del estado nutricional, pueden aparecer reacciones negativas (induración ausente $=$ PPD negativo). En casos de PPD negativo en pacientes cuyo recuento de linfocitos CD4 es $<100 / \mathrm{mm}^{3}$ conviene repetir el PPD cuando este recuento supere este nivel.

d) Quimioprofilaxis: La indicación se establece en conjunto entre el encargado del programa de TBC del servicio de salud correspondiente y el Infectólogo de referencia.

Los criterios de ingreso a la prevención son: 1. $\mathrm{PPD} \geq 5 \mathrm{~mm}$.

2. Lesiones fibróticas en radiografía de tórax (descartando TBC activa).

Como quimioprofilaxis o tratamiento de la infección tuberculosa, se recomienda el uso de Isoniazida (HIN) $5 \mathrm{mg} / \mathrm{kg} /$ día (habitualmente 300 mg) durante 9 meses, autoadministrada, pero controlada cada 15 días en su consultorio, donde se registrará la tolerancia, reacciones adversas y adherencia.
Las contraindicaciones del empleo de HIN son:

1. Antecedentes de efectos adversos severos con su uso previo

2. Enfermedad hepática severa descompensada

Las precauciones en el empleo de HIN son:

1. Interacción de fármacos: disminuye el clearance de fenitoína, carbamazepina, anticoagulantes, corticoides y acetaminofeno.

2. Dosificación según función renal: con clearance entre 10-50 dar $75 \%$ de dosis y bajo 10 dar el 50\% de dosis. En hemodiálisis administrar posterior al procedimiento.

Los encargados de TBC del servicio de salud deben llevar un registro de los pacientes VIH con quimioprofilaxis, informando al Ministerio de Salud cada 6 meses sobre su evolución (Formulario 3; Figura 2).

\section{Componente programático para pacientes con TBC}

Paciente que ingresa a programa TBC debe ser sometido a:

A) Detección de VIH (Figura 3).

a) Ofrecer test diagnóstico de infección VIH mediante consejería y consentimiento informado.

La consejería es proporcionada por el profesional de salud acreditado en los consultorios de atención primaria y debe seguir los estándares recomendados por el programa VIH/SIDA.

El test de VIH es de responsabilidad del pro- 
grama VIH/SIDA y se realiza en el consultorio bajo los estándares de confidencialidad establecidos por esta normativa y su resultado debe ser confirmado por el Instituto de Salud Pública (ISP).
Los equipos de TBC de los consultorios deben mantener un registro de pacientes que realizaron o rechazaron consejería y test diagnósticos de VIH (formulario 1; Figura 4).

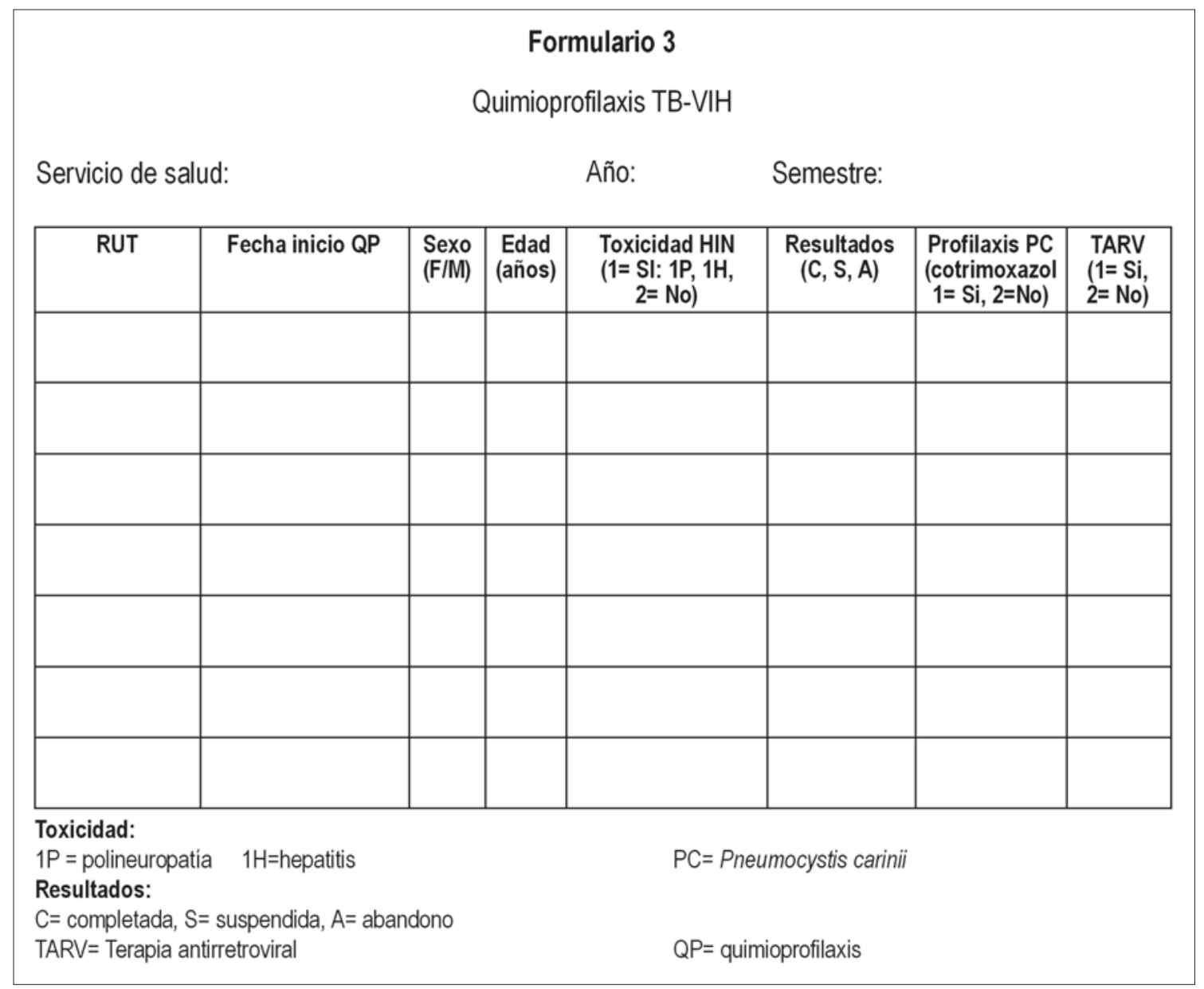

Figura 2. Formulario de Quimioprofilaxis de Tuberculosis-VIH (Formulario 3).

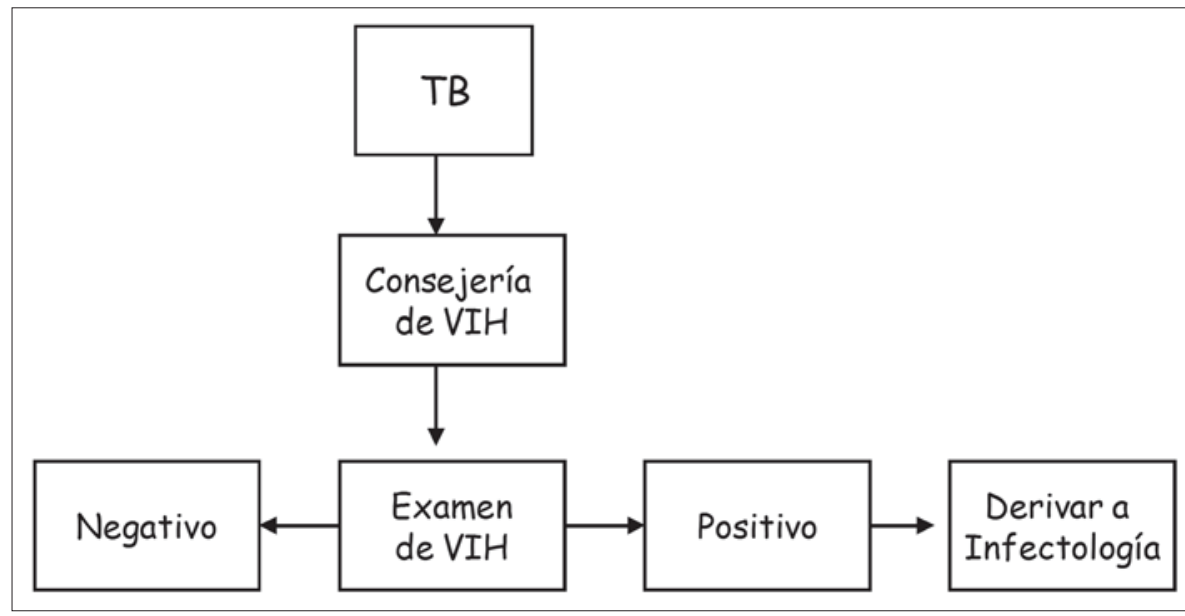

Figura 3. Flujograma del diagnóstico de VIH en tuberculosis (TB). 


\section{Formulario 1}

\section{Detección de coinfección VIH en Tuberculosis}

Servicio de salud: $\quad$ Consultorio: Año: Semestre:

\begin{tabular}{|c|c|c|c|c|c|}
\hline $\begin{array}{c}\text { Fecha inicio } \\
\text { Terapia TB }\end{array}$ & RUT & $\begin{array}{c}\text { Se solicitó } \\
\text { consejería VIH } \\
(1=\mathrm{Si}, 2=\mathrm{No})\end{array}$ & $\begin{array}{c}\text { Se realizó } \\
\text { consejeria VIH } \\
(1=\mathrm{Si}, 2=\mathrm{No})\end{array}$ & $\begin{array}{c}\text { Se realizó } \\
\text { examen } \mathrm{VIH} \\
(1=\mathrm{Si}, 2=\mathrm{No})\end{array}$ & $\begin{array}{c}\text { Resultados de VIH } \\
\text { (1= positivo; } 2=\text { negativo; } \\
3=\text { se desconoce) }\end{array}$ \\
\hline & & & & & \\
\hline & & & & & \\
\hline & & & & & \\
\hline & & & & & \\
\hline & & & & & \\
\hline & & & & & \\
\hline & & & & & \\
\hline
\end{tabular}

Figura 4. Formulario de detección de coinfección VIH en Tuberculosis (Formulario 1).

B) Estudio de susceptibilidad a fármacos antituberculosos (test de sensibilidad) y tipificación de micobacterias en ISP. Las muestras enviadas deben registrar el antecedente de "coinfección" en la solicitud de examen bacteriológico.

C) Tratamiento de la tuberculosis: La terapia debe ser administrada por personal capacitado en los consultorios de atención primaria, usando la estrategia DOTS (supervisión directa de la administración) con control del encargado de TB del servicio de salud y del infectólogo de referencia. Estos profesionales son los únicos responsables de los cambios en los esquemas y/ o duración de la terapia.

a) Según categorías:

Caso nuevo (Virgen de Tratamiento): 50 dosis diarias de Isoniazida, Rifampicina, Etambutol y Pirazinamida, seguido de 32-56 dosis bisemanales de Isoniazida y Rifampicina (dependiendo de la extensión lesional y presencia cavitaria se recomienda prolongar el tratamiento hasta 9 meses).

Antes Tratado (recaídas y abandonos): 50 dosis diarias con los mismos fármacos (pero con un agregado en la fase inicial de 25 dosis de Estreptomicina), seguido de 56 dosis bisemanales de Isoniazida, Rifampicina y Etambutol).

b) Según interacción con TARV:

Si el enfermo está recibiendo antivirales del tipo inhibidores no nucleósidos de la transcriptasa reversa (INNTR) como Efavirenz e Inhibidores nucleósidos de la transcriptasa reversa (INTR) como AZT y TC, se puede suspender la TARV en forma transitoria, por lo menos mientras dura la fase diaria de terapia de la TBC, para evitar la inducción de reacciones de reconstitución inmune (fiebre, crecimientos ganglionares, aparición de nuevos infiltrados pulmonares, etc).

Los pacientes que reciben inhibidores de proteasas (IP) y en menor grado los con INNTRV pueden ver disminuidos los niveles plasmáticos y la actividad TARV debido a la inducción del citocromo P450 hepático por parte de la Rifampicina. Además, estos fármacos producen un aumento del nivel de Rifampicina, que puede aumentar su toxicidad hepática.

En enfermos con imposibilidad de suspensión de TARV por decisión conjunta entre el encargado del programa TBC y el infectólogo de re- 


\section{Formulario 2}

\section{Casos TB-VIH}

Servicio de salud:

Año:

Semestre:

\begin{tabular}{|c|c|c|c|c|c|c|c|c|}
\hline \begin{tabular}{c|} 
Fecha inicio \\
Terapia TB
\end{tabular} & RUT & \begin{tabular}{|c|} 
Localización \\
TB \\
$(1-2-3-4)$ \\
\end{tabular} & $\begin{array}{l}\text { Sexo } \\
\text { (FIM) }\end{array}$ & $\begin{array}{l}\text { Edad } \\
\text { (años) }\end{array}$ & \begin{tabular}{c|} 
Terapia \\
(AT, VT, MDR, OE)
\end{tabular} & $\begin{array}{c}\text { Resultados } \\
(\mathrm{A}, \mathrm{Ab}, \mathrm{F}, \mathrm{Fr}, \mathrm{T})\end{array}$ & \begin{tabular}{|c|}
$\begin{array}{c}\text { Profilaxis PC } \\
\text { (cotrimoxazol } \\
1=\mathrm{Si}, 2=\mathrm{No} \text { ) }\end{array}$ \\
\end{tabular} & $\begin{array}{c}\text { TARV } \\
(1=\mathrm{Si}, \\
2=\mathrm{No}) \\
\end{array}$ \\
\hline & & & & & & & & \\
\hline & & & & & & & & \\
\hline & & & & & & & & \\
\hline & & & & & & & & \\
\hline & & & & & & & & \\
\hline & & & & & & & & \\
\hline & & & & & & & & \\
\hline
\end{tabular}

Localización TB:

1=pulmonar bacilifero $\quad 2=$ pulmonar Cultivo $(+) \quad 3=$ pulmonar sin confirmación $\quad 4=$ extrapulmonar Terapia:

$\mathrm{AT}=$ esquema para antes tratado, $\mathrm{VT}=$ esquema para virgen a terapia, MDR= esquema para multirresistente, $\mathrm{OE}=$ otros esquemas Resultados:

$A=$ alta, $A b=$ abandono, $F=$ fallecido, $F r=$ fracaso, $T=$ traslado

TARV $=$ Terapia antirretroviral

$\mathrm{PC}=$ Pneumocystis carinii

Figura 5. Formulario para casos con Tuberculosis asociada a VIH (Formulario 2).

ferencia, puede utilizarse un esquema sin Rifampicina (50 dosis diarias de Isoniazida, Pirazinamida, Etambutol y Estreptomicina, seguido de 80 dosis bisemanales de Isoniazida y Etambutol o Estreptomicina).

En los pacientes TBC asociados a VIH, se debe mantener un registro a Nivel Intermedio, por parte del encargado de TBC del servicio de salud correspondiente, informando semestralmente al Ministerio de Salud los resultados del tratamiento (Formulario 2; Figura 5).

\section{Tuberculosis Infantil}

Dr. Carlos Casar. Pediatra Broncopulmonar. Servicio de Salud Metropolitano Norte.

A pesar de la baja incidencia de TBC infantil en Chile, estos casos en niños reflejan la presencia de una fuente de transmisión adulta no reconocida o de un estudio de contactos incompleto. También estamos asistiendo a la detección de casos en hijos de inmigrantes.

Afortunadamente, el problema de la TBC infantil no es alarmante en nuestro país, pero no deseamos que más niños padezcan esta enfermedad. Esta presentación forma parte de una discusión de los especialistas pediátricos sobre las principales medidas de prevención y tratamiento infantiles.

\section{A) Prevención}

a) Vacunación con $B C G$

1. Indicaciones

- Recién nacidos con peso $\geq 2.000$ gramos al egreso de maternidad.

- Menores de 1 año no vacunados al nacer. 
- Contactos de TBC bacilíferos menores de 5 años no vacunados con BCG, después de terminar la quimioprofilaxis

2. Contraindicaciones:

- Recién nacido con menos de 2.000 gramos de peso corporal.

- Recién nacido con SIDA clínico.

- Recién nacido hijo de madre VIH (+), salvo indicación médica específica, hasta no descartar enfermedad en el niño.

- Niños con enfermedades o infecciones cutáneas extensas o que comprometan el área de vacunación.

- Enfermedades o tratamientos que condicionen inmunosupresión.

- Eritroblastosis fetal.

La cobertura de la vacunación en Chile es superior al $95 \%$ y ha logrado que no se presenten casos de meningitis TBC en niños. Sin embargo, en algún momento se planteará hasta cuando continuar con esta estrategia.

Se han propuesto los siguientes criterios para suspender la vacunación con BCG en un país:

a) Tasa anual de notificación de TBC bacilífera $<5 / 100.000$ habitantes por lo menos durante 3 años consecutivos

b) Riesgo anual de infección $<1 \%$

c) Tasa anual específica de meningitis TBC en menores de 5 años $<1 / 10.000 .000$ en los últimos 5 años.

\section{b) Quimioprofilaxis}

Consiste en la administración diaria de isoniazida $5 \mathrm{mg} / \mathrm{kg}$ durante $6-9$ meses con control médico. Se reconocen dos modalidades de quimioprofilaxis:

1. Primaria: Para niños no infectados (PPD negativo) expuestos a una fuente de contagio, con el fin de prevenir la infección TBC. Esta situación se presenta en:

a) Recién nacidos de madre tuberculosa bacilífera, que no tienen evidencias clínicas ni radiológicas de $\mathrm{TBC}$.

b) Niños no vacunados con BCG contactos de tuberculoso bacilífero, que no tengan evidencias clínicas ni radiológicas de TBC.

2. Secundaria: Para niños infectados (PPD+), con o sin BCG previo, a fin de evitar que desarrollen la enfermedad. Esta situación se presenta en:

a) Contactos intradomiciliarios de paciente con TBC bacilífera, menores de 5 años, con o sin cicatriz BCG, independiente del tamaño de la reacción del PPD (sin evidencias de enfermedad).

b) Viraje tuberculínico en un período menor de 2 años.

c) Niños de 5-14 años, contactos de bacilíferos, con PPD > $10 \mathrm{~mm}$.

d) Niños con algún grado de inmunodeficiencia.

Debemos prestar mucha atención a los niños menores de 5 años ya que todos ellos, si han estado expuestos a un paciente bacilífero, deben ingresar a quimioprofilaxis, luego de realizar PPD y radiografía de tórax. Si el PPD sigue negativo a los 3 meses de quimioprofilaxis, se puede suspender la isoniazida y vacunar al niño con BCG.

Los recién nacidos de una madre con TBC raramente requieren separarse físicamente de ella, pero deben tomarse medidas de protección incluyendo máscara de alto rendimiento NP 95 en la madre. Si ésta no es bacilífera, el recién nacido puede vacunarse, pero si es bacilífera durante el embarazo o parto, se debe descartar una TBC clínica en el niño (es importante el estudio de la placenta para descartar tubérculos). Si éste está sano clínica y radiológicamente, se debe iniciar la quimioprofilaxis y al completarla vacunarlo con BCG.

Para la interpretación del PPD se recurre a la siguiente clasificación:

Interpretación de la reacción de Tuberculina $(P P D)$

- 0 a $4 \mathrm{~mm}$ : No reactor (No infectado o falso negativo).

- 5 a 9 mm: Reactor débil (infectados TBC, reacciones cruzadas, vacunados BCG).

- 10 a 14 mm: Reactor franco (probablemente infectados, enfermos TBC, vacunados BCG, reacciones cruzadas).

- > $15 \mathrm{~mm}$ : Reactor intenso (infectados TBC o enfermos).

\section{B) Tratamiento}

El tratamiento de la tuberculosis en el niño es similar al del adulto, pero existe un esquema simplificado para el Complejo Primario Simple con sólo dos fármacos (isoniazida y rifampicina). Por otra parte, en la meningitis tuberculosa se agrega estreptomicina.

El uso de etambutol en los menores de 6 años debe ser evitado por su potencial toxicidad ocular (neuritis óptica), difícil de reconocer a esas edades (aunque se estima actualmente que no hay bases para contraindicarlo).

Los esquemas de tratamiento se especifican en las Tablas 1, 2, 3 y 4. 
Tabla 1. Tratamiento del complejo primario simple (2HR/4H2 R2)

\begin{tabular}{lcc}
\hline Droga & $\begin{array}{c}\text { Fase diaria } \\
\mathbf{5 0} \text { dosis } \mathbf{2} \text { meses } \\
\text { (10 semanas) } \\
\mathbf{~ m g / k g}\end{array}$ & $\begin{array}{c}\text { Fase bisemanal } \\
\mathbf{3 2} \text { dosis } \mathbf{4} \text { meses } \\
\text { (16 semanas) } \\
\mathbf{~} \mathbf{m g} / \mathbf{k g}\end{array}$ \\
\hline Isoniazida & 5 & 15 \\
Rifampicina & 10 & 10 \\
\hline
\end{tabular}

Tabla 3. Tratamiento de meningitis tuberculosa (2 SHRZ/4H2 R2)

\begin{tabular}{lcc}
\hline Droga & $\begin{array}{c}\text { Fase diaria } \\
\text { 50 dosis 2 meses } \\
\text { (10 semanas) } \\
\text { mg/kg }\end{array}$ & $\begin{array}{c}\text { Fase bisemanal } \\
\text { (16 semanas) } \\
\text { mg/kg }\end{array}$ \\
\hline Isoniazida & 5 & 15 \\
Rifampicina & 10 & 10 \\
Pirazinamida & 25 & - \\
Estreptomicina & 15 & - \\
\hline
\end{tabular}

*Agregar prednisona $2 \mathrm{mg} / \mathrm{kg}$, durante 2 semanas (máximo $40 \mathrm{mg}$ ), reduciendo $1 / 4$ de dosis semanalmente.

**Especialistas calificados de referencia del programa podrán prolongar duración en casos especiales.

\section{Auditorías de Muerte en Tuberculosis}

Dr. Carlos Peña. Coordinador del Comité Metropolitano de Tuberculosis.

Las muertes por TBC representan un problema. Se pierde el gran esfuerzo que se invierte en la localización de casos y se compromete la eficiencia de los tratamientos.

La magnitud de la letalidad de la TBC es sorprendente. Alrededor del 10\% de los pacientes fallece durante el tratamiento, incluso en países altamente desarrollados. Estas cifras se han mantenido en tan alta proporción durante las últimas décadas.

Para identificar las causas de este problema en Chile se realizan auditorías de todos los fallecidos por TBC. Este análisis arroja como facto-
Tabla 2. Esquema primario simplificado de tratamiento: Tuberculosis pulmonares y extrapulmonares con bacteriología negativa (2HRZ/4H2 R2)

\begin{tabular}{lcc}
\hline Droga & $\begin{array}{c}\text { Fase diaria } \\
\text { 50 dosis 2 meses } \\
\text { (10 semanas) } \\
\text { mg/kg }\end{array}$ & $\begin{array}{c}\text { Fase bisemanal } \\
\text { (16 semanas) } \\
\text { mg/kg }\end{array}$ \\
\hline Isoniazida & 5 & 15 \\
Rifampicina & 10 & 10 \\
Pirazinamida & 25 & - \\
\hline
\end{tabular}

Tabla 4. Esquema primario de tratamiento:

Tuberculosis pulmonares o extrapulmonares confirmadas bacteriológicamente y formas graves y diseminadas (2HRZE/4H2R2)

\begin{tabular}{lcc}
\hline Droga & $\begin{array}{c}\text { Fase diaria } \\
\text { 50 dosis 2 meses } \\
\text { (10 semanas) } \\
\text { mg/kg }\end{array}$ & $\begin{array}{c}\text { Fase bisemanal } \\
\text { (16 semanas) } \\
\text { mg/kg }\end{array}$ \\
\hline Isoniazida & 5 & 15 \\
Rifampicina & 10 & 10 \\
Pirazinamida & 25 & - \\
Etambutol* & 20 & - \\
\hline
\end{tabular}

*En menores de 6 años, el pediatra podrá reemplazar Etambutol por Estreptomicina.

res asociados más frecuentes: diagnóstico tardío, manejo inapropiado de la enfermedad, incluyendo falta de hospitalización oportuna, y la asociación de otras patologías o condiciones que son en realidad la causa directa de estas muertes en la mitad de los casos.

El formato de la auditoría requiere cambios en el sentido de ser más amigable y de recoger una mayor cantidad de antecedentes personales, de las diferentes patologías asociadas y de la situación social del paciente, que nos permitan diseñar mejores estrategias de prevención.

En esta presentación se incluye un formato para discutir y aplicar en fase experimental, junto con el instructivo correspondiente.

El formulario de auditoría de muerte del Programa de TBC del Minsal se muestra en la Figura 6. 


\section{Auditoría de muerte}

Servicio de salud:

Fecha:

\section{Identificación}

Nombre:

Domicilio:

\section{Tuberculosis}

Fecha notificación:

Dosis terapia:

Patología asociada:

DOTS:

\section{Auditoría}

Atención de salud:

Relación causal:

Edad:
Fecha de muerte:

Sexo:

Lugar:

\section{Localización TBC:}

RAM: MDR:

Inmunosupresión farmacológica:

Asistencia:

Fecha terapia:

Coinfección:

Control:

Acceso diagnóstico:

Observaciones:

Nombre Auditor:

Figura 6. Formulario de Auditoría de muerte, Programa Nacional de Control de la Tuberculosis. MINSAL. RAM: reacciones adversos a medicamentos. MDR: multirresistencia confirmada. DOTS: Supervisión directa de la administración.

\section{Instructivo de auditoría de muerte en tubercu-} losis, Programa de Control de la TBC, Minsal

1. La auditoría debe ser realizada por el encargado de tuberculosis del servicio de salud (nivel intermedio).

2. El encargado de tuberculosis debe seleccionar las auditorías:

Se incluyen:
a) Todos los fallecidos intratratamiento in- dependiente de la causa.
b) Las personas cuyos certificados de de- función señalen a la tuberculosis como la causa principal o asociada de muerte aun- que no se encuentren en tratamiento.

Se excluyen:
a) Los certificados que indican a la tubercu- losis como una secuela tardía no relacio- nada con la muerte.
b) Las micobacterias atípicas.

3. La edad se expresa en años y el sexo en $\mathrm{M}=$ masculino $\mathrm{y} \mathrm{F}=$ femenino.

4. El domicilio es el habitual o donde vivía el último mes antes de morir.

5. La fecha de muerte es la oficial del certificado de defunción.

6. El lugar de ocurrencia se clasifica en:
1) Domicilio.
2) Vía pública.
3) Hospital.
4) Servicio de urgencia o posta.

7. La fecha de notificación se extrae del Bole- tín de enfermedades de notificación obligatoria, si no está notificado se coloca: SN.

8. La localización de la tuberculosis se registra como:

1) Pulmonar bacteriología positiva (baciloscopia o cultivo de Koch).

2) Pulmonar no bacteriológica.

3) Meníngea.

4) Miliar.

5) Osteoarticular.

6) Urogenital.

7) Pleural.

8) Diseminada (2 ó más localizaciones o localizada con hemocultivo positivo para micobacterias).

9) Otras localizaciones (señalar).

9. La fecha de inicio de terapia se expresa en día-mes-año.

Si no recibió terapia se coloca: $\mathrm{ST}$.

10. El tipo de terapia se consigna como:

1) Esquema primario (2HRZE/4H2R2).

2) Esquema primario prolongado (igual a 1 pero con mayor número de dosis).

3 ) Esquema primario simplificado (2HRZ/ 4H2R2).

4) Esquema de antes tratado (1SHRZE/ 1HRZE/7H2R2).

5) Esquema multirresistente.

6) Otro esquema diferente (señalar).

11. Las dosis de terapia de tuberculosis se colocan como: número de dosis diarias y número de dosis intermitentes (“x” DD + “y” DI). 
12. Las reacciones adversas a los fármacos (RAM) antituberculosos se consignan como:

1) Anafilaxia (alergia sistémica severa).

2) Reacción cutánea.

3) Daño hepático agudo.

4) Neurológico (convulsiones, polineuropatía).

5) Neuritis óptica.

6) Daño hematológico (anemia, trombocitopenia, leucopenia, aplasia medular).

7) Articular (artralgias, gota, algodistrofia).

8) Daño renal.

9) VIII par (hipoacusia, vértigo).

10) Gastritis, reflujo GE o úlcera GD.

11) Otras reacciones (señalar).

12) Se desconoce.

13) No informadas.

13. MDR se refiere a la existencia de multirresistencia confirmada con el estudio de susceptibilidad a fármacos $(\mathrm{H}+\mathrm{R})$. Se contesta como:

1) No.

2) Sí.

3) Se desconoce.

14. La coinfección se refiere a la existencia de serología confirmada a VIH y se contesta como:
1) No.
2) Sí.
3) Se desconoce.

15. La inmunosupresión no debida al VIH considera el uso hasta 3 meses antes de la muerte de terapia con inmunomoduladores en colagenopatías y trasplantes, quimioterapia antineoplásica, fármacos inmunosupresores incluidos pednisona más de $20 \mathrm{mg} /$ día por más de 1 mes y se considera como:

1) No hay inmunosupresión por fármacos.

2) Existe inmunosupresión por fármacos.

3) Se desconoce.

16. La patología asociada se consigna como:

1) Neoplasia.

2) Cardiopatía.

3) Asma o EPOC.

4) Diabetes.

5) Neuropatía.

6) Neurológico (incluye AVC).

7) Colagenopatía.

8) Otras (señalar).

17. DOTS es la terapia controlada bajo observación directa y suministrada por personal de salud capacitado (en consultorio, domicilio y hospitales). Contestar:

1) DOTS adecuado.
2) DOTS dudoso.

3) Sin DOTS.

18. La asistencia a terapia se refiere a la fase diaria o intermitente. Consignar:

1) Asistencia regular (sólo una ausencia al mes).

2) Asistencia irregular (más de una ausencia por mes).

3) Inasistencias (inasistente hasta por una semana).

4) Abandono (inasistencia de un mes o más).

19. El control de la terapia se refiere al control por médico en casos ambulatorios y se consigna como:

1) Con control médico mensual.

2) Sin control médico mensual.

20. La atención de salud son prestaciones otorgadas por profesional de la salud en consultorios, centros de especialidades, servicios de urgencia o centros hospitalarios y se consigna como:

1) Atención médica antes de una semana del fallecimiento.

2) Atención médica entre una semana y un mes antes de fallecer.

3) Atención médica hace más de un mes.

4) Sin atención médica.

5) Se desconoce.

21. El acceso al diagnóstico se califica como:

1) Registra sólo una consulta antes del diagnóstico.

2) Registra 2 ó más consultas el último mes antes del diagnóstico.

22. La oportunidad de diagnóstico se califica como:

1) Menos de un mes de síntomas respiratorios antes del diagnóstico en los casos pulmonares con bacteriología positiva.

2) Más de un mes de síntomas respiratorios antes del diagnóstico en los casos pulmonares con bacteriología positiva.

23. La relación causal se refiere a la estimación de la contribución de la tuberculosis en la causa de fallecimiento y se consigna como:

1) La tuberculosis es la causa directa de muerte.

2) La tuberculosis no es la causa directa de muerte, pero contribuye a ella.

3) La tuberculosis no incide en la causa de muerte.

24. En observaciones se colocan aspectos no considerados en este instructivo y que el evaluador requiere precisar. 